\title{
Continuum models and Morse Theory in the simulation, design and evaluation of magnetic Skyrmion devices
}

\author{
P. Robert Kotiuga ${ }^{1}$, , Member, IEEE \\ ${ }^{1}$ Dept. of ECE, Boston University, Boston, MA 02215, USA
}

\begin{abstract}
Nanomagnetic devices such as computer gates and memory devices based on magnetic skyrmions are close to becoming a reality. In this paper we will explore the hghly nonconvex nanomagnetic energy landscape in order to draw conclusions about the complexity of magnetic phenomena. Morse theoretic arguments show that, in a bounded energy interval, the number of critical points of the energy functional grows exponentially. To show this, we introduce a hierarchy of models for the design of nanomagnetic devices to provide a solid foundation for the introduction of topological tools. To reason in terms of lattice models, one must make a distinction between two types of lattices: the quantum mechanical model of the actual physical lattice and the lattice model that can be associated with a discretization of a continuum model of the physics. By focuing on the implications of Morse theory applied to lattice systems arising from the discretization of the continuum models, and the notion of "topological frustration", we provide a framework for understanding "complexity" in the context of nanomagnetic systems. We conclude with some suggestions for making the analysis more qualitative.
\end{abstract}

Index Terms-Topological Phenomena, Nanomagnetics, Information Storage, Magneto-Electronics, Spin Electronics,

\section{INTRODUCTION}

C OMPUTER gates and memory devices based on magnetic skyrmions are close to becoming a reality [1]. Skyrmion-based electronic devices are a subset of spintronic nanoscale devices based on chiral materials [2]. [3]. The Dzyaloshinskii-Moriya (D-M) interaction is a chiral magnetic interaction which models chiral magnetic materials showing particular promise for extending CMOS compatible Skyrmion electronics at, or below, $5 \mathrm{~nm}$ where silicon devices are no longer competitive [3]. This technology is a natural extension of the device physics underlying MRAM [4], [5]. The models used for nanoscale device design are phenomenological in nature and there are problems interpreting both boundary conditions and limiting conditions associated with singularities.

The purpose of this paper is to introduce a hierarchy of models for the design of such devices and to provide a solid foundation for the introduction of topological tools; specifically, Morse theory. The models are:

1) A lattice model which is a model of the physics of the physics at the atomic level. Very little analysis is performed at this level other than to observe that the exchange coupling leads to an elliptic system of integrodifferential equations associated with the Hamiltonian when a continuum model is made.

2) A continuum model which is used in the LLG equation. Regularity results about the elliptic principal symbol dictate the possibilities for topological phenomena associated with defects. It is only at this level that topological arguments can be made and a proper formulation of boundary conditions can be formulated.

3) Lattice systems associated with discretizations of the continuum model. Although these models can be interpreted in physical terms, they are not a substitute for the physics that can be formulated in terms of

Manuscript received ... , 2021. Corresponding author: P. Robert Kotiuga (email: prk@bu.edu ). the actual quantum physics associated with the atomic lattice. However, the existence of a continuum limit enables one to use relatively crude discretizations while having confidence that, when refined, they model the physcs of the atomic lattice in a meaningful way.

We show that the interplay between these three types of models leads to geometric and topological formulations of issues associated with defects and boundary conditions [6], a clearer understanding of the use of the LLG equation in the nanoscale devices, as well as a more geometric connection to the mathematical formalism used to describe quantum phenomena. In the process, the role of chirality emerges more cleanly and it points to the role of topology in the possibility of near reversible computing generating a minimum of entropy and heat [7], [8], [9]. By focuing on the implications of Morse theory applied to lattice systems arising from the discretization of the continuum models, and the notion of "topological frustration", we provide a framework for understanding "complexity" in the context of nanomagnetic systems.

\section{ON THE USE OF LATTICE MODELS}

That nanomagnetic devices are designed and simulated by using the LLG formalism of micromagnetics with spin-torque corrections, indicates that the underlying mathematical models used are largely phenomenological; lattice models are used in a rather ad hoc manner as long as they enable experimentalists to successfully fit mathematical formulas to experimental data while avoidng inessential theoretical aspects of the underlying physical phenomena. However seductive reasoning in terms of such lattice models may be, one must remember that they are not based in the actual physical lattice of magnetic spins, but a coarser lattice which can be associated with a discretization of a continuum theory. Unfortunately, for summarizing "topological aspects" of experimental research, lattice models are meaningless if they cannot be derived from basic principles arising from the notion of continuity. 
In principle, the topological formulae should follow from the hypotheses that justify the assumption of a continuum model. In micromagnetics, the hypothesis of continuity comes from an analysis of the quantum mechanical exchange coupling in the physical lattice. So in the first pass, if one is to reason in terms of a lattice model, one must make a distinction between two types of lattices:

- A quantum mechanical model of the actual physical lattice, and

- A lattice model that can be associated with a discretization of a continuum model of the phenomena.

It is only in this latter case, that topological formulae can be derived from more basic physical principles. In this paper we will explore the hghly nonconvex energy landscape in order to draw conclusions about the "complexity" of magnetic phenomena by showing that, on a interval where the energy is bounded, the numer of critical points of the energy functional grows exponentially. To do this, recall that the micromagnetic energy functional is a sum of four terms:

$$
U_{M a g}=U_{\text {exch }}+U_{\text {anis }}+U_{\text {demag }}+U_{\text {ext }},
$$

where the subscripts on the energy contributions refer to echange, anisotropy, demagnetization and external.

Consider a lattice with $N$ sites, and upper and lower bounds for the various energy terms as a function of $N$. Here, $N$ is the total number of sites, so that if one has a three dimensional lattice with $n_{i}$ sites in the $i^{t h}$ of three mutually perpendicular directions, then $N=n_{1} n_{2} n_{3}$. It is easy to derive upper and lower bounds on $U_{a n i s}$ and $U_{\text {ext }}$ which depend on the volume of the lattice (which remains unchanged under a refinement of the lattice), but not on $N$. To derive upper and lower bounds for $U_{\text {exch }}$ and $U_{\text {demag }}$, it is useful to constider two extreme configurations of magnetization: the constant magnetization, which maximizes $U_{\text {demag }}$ and minimizes $U_{\text {exch }}$, and a three-dimensional checker board pattern minimizes $U_{\text {demag }}$ and maximizes $U_{\text {exch }}$. The values of $U_{\text {demag }}^{\text {min }}$ and $U_{\text {exch }}^{\text {min }}$ are both close to zero, but their maxima have a very different dependence on $N$; $U_{\text {demag }}^{\max }$ depends on the volume of the sample, but not on $N$. On the other hand, $U_{\text {exch }}^{\max }$ depends on the volume of the sample, but also on $N$ since finer discretizations can model finer checker board patterns so, neglecting lower order terms associated with the boundary of the sample,

$$
U_{\text {exch }}^{\max }=a_{0} E_{0} N
$$

Here $E_{0}$ depends on the exchange coupling at a site in the atomic lattice, and $a_{0}$ is a dimensionless constant that depends on the ratio volume of the sample to the volume of the cell in the atomic lattice.

Although the above arguments were phrased in terms of the individual contributions to the magnetic energy, $U_{M a g}$, they can be used to obtain simple upper and lower bounds on $U_{\text {Mag }}^{\max }$, and $U_{\text {Mag }}^{\min }$, as well as the estimate for

$$
U_{\text {Mag }}^{\max }-U_{M a g}^{\min }=a E_{0} N+b
$$

where $a$ and $b$ are positive constants and an upper bound for $a$ can be given as a function of $a_{0}$ which is close to linear. The key point of this crude estimate is that it is linear in $\mathrm{N}$.
The use of Morse theory in the remainder of this paper will show that, between the energies associated with the minimum and maximun energy configurations for the magnetization on a lattice with $N$ spins, there are a minimum of $2^{N}$ critical points for the energy functional. This shows that, given some initial configuration of magnetization the dynamics of the system are generically incredibly chaotic. Furthermore, this conclusion will hold for LLG dynamics, with or without spintorque corrections, or any other dynamics that decrease the overall energy of the system! Note that this conclusion cannot be obtained from the continuum limit by taking a naive limit as $N$ tends to infinity. On the other hand, the existence of the continuum micromagnetic model is essential for making sense of the refinements of crude lattice approximations.

\section{EXCHANGE ENERGY AND THE ROLE OF ELLIPTICITY IN CONTINUUM MODELS}

In a summary of the operation of a nanomagnetic device, mathematical formulae are often substituted for an awkward sentence; they describe the phenomena but no argument is made to show that the equations should be a logical consequence of some simple assumptions (e.g. continuity etc.) To make the connection back to physics, one has to introduce the notion of quantum mechanical exchange energy which tries to align spins so that over small distances the orientations of the spins do not change quickly. In the isotropic case, the exchange terms reduce to either a Laplacian or a D$\mathrm{M}$ interaction. Because it takes considerable mathematical overhead to introduce topological formalism at a fundamental level, there is a tendency to reduce concepts like Skyrmions to a taxonomy of suggestive, highly symmetric, but incomplete pictures, and treat the equations like cut and paste quotations from a foreign language. In order to think beyond equations we need to put some trust in the notion of an elliptic partial differential equation. Basically, elliptic equations are like ordinary differential equations in the sense that their solutions are much smoother than the boundary values or "excitations" in the domain. This is in stark contrast to the 1-D wave equation which admits discontinuous right and left going solutions, even though the coefficients of the eauation are infinitely differentialble.

In more precise language we can say that the underlying continuum model of the micromagnetic phenomena models the exchange energy as a differential operator having an elliptic principal symbol, and the topological formulae follow from standard arguments of algebraic topology and elliptic regularity theory applied to the exchange energy term. Without these mathematical arguments, the presentation of formulas of a topological nature is necessarily opaque. In micromagnetics the richness of topological phenomena then follows from the order parameter space being the sphere. From a mathematical point of view, there are some parallels with quasistatic electromagnetic phenomena where Kirchhoff's view of circuit theory comes form topological degrees of freedom surviving in the low-frequency limit of Maxwell's equations via the elliptic equations associated with Hodge theory[12].

In principle, one could obtain the results of this paper by applying Morse theory to a continuum problem but, because 
of the pointwise constraint on the norm of the magnetization vector, one would obtain a nonlinear eigenvalue problem for a vector integro-differential equation whose principal symbol is elliptic. However the Lagrange multiplier corresponding to the constraint is not an eigenvalue, but an eigenfield which varies from point to point in space, and it is very difficult to get an explicit handle on the spectrum for the purpose of quantifying the type of density of states argument we need. For this reason, we present Morse theory in the context of the discretized systems.

\section{MORSE THEORY APPLIED TO DISCRETIZATIONS; TOPOLOGICAL FRUSTRATION}

"Topology! The stratosphere of human thought! In the twenty-fourth century it might possibly be of use ..."-Aleksandr Solzhenitsyn, The First Circle

There are many excellent sources for Morse theory in finite dimensions, from classic texts that that build on the basics of multivariable calculus [10], to more recent books that stress the connection to physics [11]. The basic result in the field are the Morse inequalities; they are summarized in Appendix A. Morse theory in infinite dimensions finds a natural home in the calculus of variations. Although we can associate the exchange energy term with a harmonic map for the purpose of introducing Morse theory, in the absence of singularities forced by topological constraints, one can use lattice models to inform the study of the continuum model. In particular, one can apply Morse theory to the lattice models of harmonic maps and exchange energy.

The key observation for applying the Morse inequalities is to realize that the configuration space for a lattice with $\mathrm{N}$ sites is the Cartesian product, denoted $\times$, of $\mathrm{N}$ copies of $S^{2}$; the unit sphere in three dimensional Euclidean space. That is, if $\mathcal{C}_{N}$ is the configuration space of the micromagnetic system,

$$
\mathcal{C}_{N}=S^{2} \times S^{2} \times S^{2} \times \ldots \times S^{2}, N \text { times }
$$

Furthermore, the Poincaré polynomial for the Cartesian product of two spaces is the product of the Poincaré polynomials of the two spaces. That is,

$$
P_{t}\left(M_{1} \times M_{1}\right)=P_{t}\left(M_{1}\right) P_{t}\left(M_{2}\right)
$$

(For the record, this is a corollary of the so called Kuneth formula that states that cohomology algebral of the Cartesian product of two spaces is the free product of the the cohomology algebras of the individual spaces.) Note that this formula can be iterated to obtain the Poincaré polynomial for repeated Cartesian product of spaces.

Putting the above two equatons together and knowing that the Poincaré polynomial for the sphere is just $\left(1+t^{2}\right)$, we have that,

$$
P_{t}\left(\mathcal{C}_{\mathcal{N}}\right)=\left(P_{t}\left(S^{2}\right)\right)^{N}=\left(1+t^{2}\right)^{N}
$$

Since Morse functions are dense in the space of functions, without loss of generality, we can consider and micromagnetic energy function $U_{M a g}$ to be a Morse function. The Morse inequalities then become,

$$
\mathcal{M}_{t}\left(U_{M a g}\right)-P_{t}\left(\mathcal{C}_{N}\right)=(1+t) Q_{t}\left(U_{M a g}\right)
$$

or,

$$
\mathcal{M}_{t}\left(U_{M a g}\right)=\left(1+t^{2}\right)^{N}+(1+t) Q_{t}\left(U_{M a g}\right)
$$

From this, there are two immediate conclusions we can draw:

1) Although the Morse inequalities don't say anything useful about the critical points of $U_{M a g}$ which have odd index, the number of critical points of index $2 k$ are bounded below by the binomial coefficient,

$$
m_{2 k}\left(U_{M a g}\right) \geq\left(\begin{array}{c}
2 N \\
2 k
\end{array}\right)=\frac{(2 N) !}{(2 k) !(2(N-k)) !} .
$$

To appreciate the size of this lower bound for "middle dimensional" critical points whose index is $\mathrm{N}$, we can assume $N$ is even, set $k=N$ in this expression, and use Stirling's approximation to find:

$$
m_{N}\left(U_{M a g}\right) \geq\left(\begin{array}{c}
2 N \\
N
\end{array}\right)=\frac{(2 N) !}{((N) !)^{2}} \simeq \frac{2^{N}}{(\pi N)^{\frac{1}{2}}} .
$$

2) Setting $t=1$ in the Morse inequalities above, we get a lower bound on the total number of critical points of $U_{M a g}$ :

$$
\sum_{k=0}^{2 N} m_{k}\left(U_{M a g}\right)=\mathcal{M}_{1}\left(U_{M a g}\right)=2^{N}+2 Q_{1}\left(U_{M a g}\right)
$$

or, since $Q$ is a polynomial with positive coefficients,

$$
\sum_{k=0}^{2 N} m_{k}\left(U_{M a g}\right) \geq 2^{N}
$$

These two Morse-theoretic observations can be divided by the energy bound estimates of section 2 to obtain density of staes statements reminiscent of the physics literature:

1) For the estimate involving critical points of even index,

$$
\frac{m_{2 k}\left(U_{M a g}\right)}{U_{M a g}^{\max }-U_{M a g}^{\min }} \geq \frac{\left(\begin{array}{c}
2 N \\
2 k
\end{array}\right)}{a E_{0} N+b},
$$

and for "middle dimensional" critical points whose index is even and close to $\mathrm{N}$ :

$$
\frac{m_{N}\left(U_{M a g}\right)}{U_{M a g}^{\max }-U_{M a g}^{\min }} \geq \frac{\left(\begin{array}{c}
2 N \\
N
\end{array}\right)}{a E_{0} N+b} \simeq \frac{2^{N}}{a E_{0} \pi^{\frac{1}{2}} N^{\frac{3}{2}}} .
$$

2) From the lower bound on the total number of critical points of $U_{M a g}$, we have,

$$
\frac{\sum_{k=0}^{2 N} m_{k}\left(U_{M a g}\right)}{U_{M a g}^{\max }-U_{M a g}^{\min }} \geq \frac{2^{N}}{a E_{0} N+b}
$$

It is now apparent that the simple upper and lower bounds on the discretized exchange energy derived in section two, along with the consequence for upper and lower bounds on $U_{M a g}$, when combined with Morse-theoretic considerations, show that the number of critical points lying within a fixed energy range has an exponential dependence on the number of lattice points in a discretization. This is quite independent of the physical lattice associated with the magnetic material. The author refers to this as "topological frustration and there are ties to the notions of chaos and phase transitions. For the numerical analyst, these arguments can be refined considerably 
by bringing more detail about the energy functional; the simple hypothesis that the energy of the "demag field" is maximum when the exchange energy in minimum, and vice versa should be refined by tighter upper and lower bounds on $U_{M a g}$. Considering families of lattice models associated with the discretization of continuum models we now have a tie to the error analysis in numerical simulations. To get a better physical feel for the implications of these estimates, we need to bring more detailed information about ability of the discretization to resolve the structure of domain walls, the level of the Curie temperature, and thermal consideratins involving $k T$.

\section{Conclusions}

In this paper we will explored the hghly nonconvex nanomagnetic energy landscape in order to draw conclusions about the complexity of magnetic phenomena. This is essential for understanding the dynamical systems arising in the simulation of nomagnetic devices such as computer gates and memory devices based on magnetic skyrmions. Morse theoretic arguments were used to show that, in a bounded energy interval, the numer of critical points of the energy functional grows exponentially. In order to to frame the Morse theoretic arguments, we introduced a hierarchy of models for the design of nanomagnetic devices to provide a solid foundation for the introduction of topological tools. To reason in terms of lattice models, we made a distinction between two types of lattices: the quantum mechanical model of the actual physical lattice and the lattice model that can be associated with a discretization of a continuum model of the physics. In secion two of the paper, simple upper and lower energy bounds which were used in section four to reinterpret the Morse theoretic argument in terms of density of states. In section four, the systematic use of Morse theory in the context of the configuration space of a magnetic material appears to be qute unprecidented in the liturature. By focuing on the implications of Morse theory applied to lattice systems arising from the discretization of the continuum models, and the notion of topological frustration, we provide a framework for understanding complexity in the context of nanomagnetic systems. We conclude with some suggestions for making the analysis more qualitative. The basics of the Morse inequalities are reviewed in Appendisx A.

\section{APPENDIX A}

\section{THE MORSE INEQUALITIES}

Morse theory in finite dimensions starts with a compact orientable n-dimensional manifold $M$. Let the $i^{\text {th }}$ Betti number (i.e. the rank of the $i^{\text {th }}$ homology group) be denoted by $\beta_{\mathrm{i}}(\mathrm{M})$. Roughly speaking, it counts the number of ways an $i$-dimensional cycle is not the boundary of any $(i+1)$ dimensional domain. The generating function for the Betti numbers, known as the Poincaré polynomial is key to our discussion:

$$
P_{t}(M)=\sum_{k=0}^{n} t^{k} \beta_{k}(M)
$$

Consider a function $f$, mapping $\mathbf{M}$ to $\mathbf{R}^{1}$. The critical points of $f,\left\{p_{i}\right\}$, are said to be nondegenerate if the Hessian matrix (i.e. the symmetric matrix of second partial derivatives) has full rank at every critical point. A function with a finite number of isolated critical points, all of which are nondegenerate, is called a Morse function. Morse functions are abundant in the sense that they form an open dense set in the space of all functions. That is, any function can be approximated arbitrarily closely by a Morse function.

Given a Morse function on $M$, we define the index of the $j^{\text {th }}$ critical point, $\mathbf{p}_{j}$, to be the number of negative eigenvalues of the Hessian matrix evaluated at $p_{j}$. (Note: the eigenvalues are real because the Hessian is symmetric, and nonzero since $p_{j}$ is nondegenerate.) Let $m_{i}(f)$ be the number of critical points of index $\mathrm{i}$, and define the Morse polynomial of $f$, by the generating function:

$$
\mathcal{M}_{t}(f)=\sum_{k=0}^{n} t^{k} m_{k}(f)
$$

The fundamental result of Morse theory, called the Morse inequalities, asserts that

$$
\mathcal{M}_{t}(f)-P_{t}(M)=(1+t) Q_{t}(f)
$$

where $\mathrm{Q}(\mathrm{f})$ is some polynomial with nonnegative integer coefficients. A quick corollary of this result are the weak Morse inequalities:

$$
m_{k}(f) \geq \beta_{k}(M)
$$

An equivalent formulation of statement of the Morse inequalities in terms of generating functions, are the strong Morse inequalities. They are found by equating the coefficients of powers of $t$, solving a triangular linear system for the coefficients of $\mathrm{Q}_{t}(\mathrm{f})$ and using the nonnegativity of these coefficients to obtain the inequalities:

$$
\begin{aligned}
m_{0} & \geq \beta_{0} \\
m_{1}-m_{0} & \geq \beta_{1}-\beta_{0} \\
m_{2}-m_{1}+m_{0} & \geq \beta_{2}-\beta_{1}+\beta_{0} \\
\vdots & \geq \quad
\end{aligned}
$$

The Morse Inequalities, expressed in terms of generating functions, suggest two strategies for their use:

1) If $P_{t}(M)$ is known, then one has a lower bound of the number of critical points of every index. Specifically, a very loose bound is given by $m_{i} \geq \beta_{i}$ for all $i$.

2) If no consecutive powers of $t$ occur in the Morse polynomial then $Q_{\mathrm{t}}(f)=0$. In this case the Inequalities become equalities and $\mathcal{M}_{t}(f)=P_{\mathrm{t}}(M)$. In this case knowledge of the critical points gives complete knowledge of the Betti numbers.

A function for which $\mathcal{M}_{\mathrm{t}}(\mathrm{f})$ is equal to $\mathrm{P}_{\mathrm{t}}(\mathrm{M})$ is called a perfect Morse function. In general, there are topological obstructions to finding a perfect Morse function. For example, the Morse inequalities are still valid if homology groups are computed using coefficients from some other number field (e.g. $\mathbb{Z}_{\mathrm{p}}$ where $\mathrm{p}$ is a prime). 


\section{REFERENCES}

[1] K. Everschor-Sitte, J. Masell, R. M. Reeve , and M. Kläui, Magnetic skyrmions-Overview of recent progress in an active research field, J. Appl. Phys. 124, 240901 (2018); https://doi.org/10.1063/1.5048972

[2] Kang, W., Huang, Y., Zhang, X., Zhou, Y., Zhao, W., "SkyrmionElectronics: An Overview and Outlook", Proc. IEEE, vol. 104, no.10, Oct 2016; pp 2040-2061.

[3] Sch"utte,C., Rosch, A., "Dynamics and energetics of emergent magnetic monopoles in chiral magnets", Phys. Rev. B, vol. 90, 174432 (2014).

[4] Engel-Herbert, R., Locatelli, A., Cherifi, S., Schaadt, D. M., Mohanty, J., Ploog, J.H., Bauer, E., Belkhou, R., Heun, S., Pavlovska, A., Leo, T., Hesjedal, T., "Investigation of magnetically coupled ferromagnetic stripe arrays", Applied Physics A, vol 84, 2006, pp 231-236, DOI: 10.1007/s00339-006-3619-8

[5] Kazemi, M., Rowlands, G.E., Ipek, E., Buhrman, R.A., Friedman, E.G., "Compact Model for spin-Orbit magnetic Tunnel Jubnctions." IEEE Trans ELECTRON DEV, vol. 63, no. 2, FEB.2016, pp 848-855.

[6] Kotiuga, P. R., "Weitzenbock Identities and Variational Formulations in Nanophotonics and Micromagnetics". IEEE Trans. MAG, vol. 43, no. 4, April 2007, pp1669-1672.

[7] Kotiuga, P.R., "The Algebraic Topology of Bloch Points", IEEE Trans. MAG, vol. 25, no. 5, Sept. 1989, pp. 3476-3478.

[8] Kotiuga, P.R., Toffoli, T., "Potential for Computation in Micromagnetics via Topological Conservation Laws", Physica D, vol. 120, 1998, pp. 139-161.

[9] Kotiuga, P.R., Giles, R.C., "A Topological Invariant for the Accessibility Problem of Micromagnetics", Journ. Applied Phys., vol. 67 no. 9, May 1990, pp. 5347-5349.

[10] Milnor, John. "Morse Theory". (AM-51), Princeton: Princeton University Press, 2016. https://doi.org/10.1515/9781400881802

[11] Yukio Matsumoto, "An Introduction to Morse Theory". Translated by Masahico Saito and Kiki Hudson, Translations of Mathematical Monographs, Vol. 208; AMS, 2002. https://doi.org/10.1090/mmono/208

[12] P.W. Gross, P.R. Kotiuga, "Electromagnetic Theory and Computation: A Topological Approach". MSRI Monograph series, Vol. 48; Cambridge U. Press, 2004. ISBN 0521 801605. See ch. 1 and app. MA

P. Robert Kotiuga received his B.Eng., M. Eng., and Ph.D. degrees from McGill University in 1981, 1982, and 1985 respectively. After a post-doc at MIT, he joined Boston University in 1987. Over the years he has held visiting appointments at MIT (Cambridge MA), ETH (Zurich), U. Pau (France), TUT (Finland), Univ of Trento (Italy), and other shorter appointments.

Prof. Kotiuga's research focuses on topological aspects of 3-dimensional problems in computational electromagnetics, the use of Whitney forms and simplicial data structures in the context of the finite element method. His earlier work on cuts for magnetic scalar potentials and helicity functionals has, in recent years, led to a topological characterization of near force-free magnetic fields. His early topological work in the context of vertical Bloch line memories now informs topological considerations in nanoscale MRAM and Skyrmion devices. More recently, informed by psychoacoustics, he is revisiting issues of transient modeling in electroacoustics.

Two books tied to his research are: Gross, P.W., Kotiuga, P.R., Electromagnetic Theory and Computation: A Topological Approach. (Cambridge U. Press, 2004), and Kotiuga, P. R., (editor), A Celebration of the Mathematical Legacy of Raoul Bott, (CRM Proceedings and Lecture Notes, Vol. 50, AMS, 2010.) 